\title{
New dry electrodes based on iridium oxide (IrO) for non-invasive biopotential recordings and stimulation
}

\author{
N.S. Dias ${ }^{\mathrm{a}, \mathrm{b}, *}$, J.P. Carmo ${ }^{\mathrm{a}}$, A. Ferreira da Silva ${ }^{\mathrm{a}}$, P.M. Mendes ${ }^{\mathrm{a}}$, J.H. Correia ${ }^{\mathrm{a}}$ \\ a University of Minho, Department Industrial Electronics, Campus Azurem, 4800-058 Guimaraes, Portugal \\ b School of Technology, Polytechnic Institute of Cávado and Ave, 4750-810 Barcelos, Portugal
}

\section{A R T I C L E I N F O}

\section{Article history:}

Received 27 May 2010

Received in revised form 10 August 2010

Accepted 18 September 2010

Available online 29 September 2010

\section{Keywords:}

Biopotential acquisition

Stimulation

Dry electrode

Microtips array electrode

Iridium oxide ( $\mathrm{IrO}$ ) electrode

Micromachining

Sputtering

Thin-films

\begin{abstract}
A B S T R A C T
This paper presents a new type of dry electrodes for acquisition of biopotentials and stimulation. These dry electrodes are composed by 16 microtip structures (by forming an array of $4 \times 4$ microtips), which were fabricated through bulk micromachining of a $\langle 100\rangle$-type silicon substrate in a potassium hydroxide $(\mathrm{KOH})$ solution. The fabrication process was trimmed in a way that each microtip presents solid angles of $54.7^{\circ}$, a width in the range $150-200 \mu \mathrm{m}$, a height of $100-200 \mu \mathrm{m}$, and an inter-microtip spacing of $2 \mathrm{~mm}$. The electrodes have a thin layer (obtained by reactive DC-sputtering) of iridium oxide (IrO) to improve the contact with the skin. These dry electrodes penetrate the outer skin layer (i.e. stratum corneum that is $10 \mu \mathrm{m}$ thick) to allow a direct contact with the electrolyte fluids of the inner skin layers. The new electrode avoid the use of conductive gels and reduce the skin preparation time in EEG experiments, which may take about $45 \mathrm{~min}$ for a set of 32 standard silver/silver chloride $(\mathrm{Ag} / \mathrm{AgCl})$ electrodes. The electrode-electrolyte impedance spectrometry (IS) of the IrO thin-films was performed in a saline solution, $0.9 \%$ concentration by weight, to mimic the electrode-tissue interface. The IS measured results for the IrO coatings were comparable to the results observed for the standard $\mathrm{Ag} / \mathrm{AgCl}$ electrodes. The new dry microtips array constitutes an inexpensive, low resistance and mechanically robust alternative electrode for non-invasive biopotential recording/stimulation with fast application on skin.
\end{abstract}

() 2010 Elsevier B.V. All rights reserved.

\section{Introduction}

Recent advances in the biomedical field related with medicine and biology have been demanding more sophisticated electrode fabrication technologies [1]. Electrical activity occurs between neurons as well as in the muscles (e.g. heart) and nerves. The biopotential electrodes, jointly with acquisition systems, sense the electrical activity and make it accessible for clinical and research trials. Electrodes may also be employed on stimulation of excitable tissue.

Biopotential recording and excitable tissue stimulation have been accomplished by recurring to invasive and non-invasive electrodes. The neuroscience field has been demanding invasive electrodes that are implanted for single and multiple recording sites, deep brain stimulation (DBS) and alleviate symptoms of Parkinson's disease [2]. Non-invasive electrodes are used for biopotential recordings like ElectroEncephaloGram (EEG) [3], ElectroOculoGram (EOG), ElectroCardioGram (ECG), ElectroMyoGram (EMG), among several other signals from the skin surface. The surface functional electrical stimulation (FES) [4] and electrotactile stimulation [5] is also accomplished by non-invasive electrodes.

\footnotetext{
* Corresponding author. Tel.: +351253 510190; fax: +351253510189.

E-mail address: ndias@dei.uminho.pt (N.S. Dias).
}

Different electrode materials have been tested on biopotential recording systems: silver/silver chloride $(\mathrm{Ag} / \mathrm{AgCl})$ [6], titanium nitride (TiN) [7], aluminum ( $\mathrm{Al}$ ) [8], platinum (Pt) [9] and iridium oxide (IrO) [10]. Standard sintered $\mathrm{Ag} / \mathrm{AgCl}$ ring electrodes are frequently used for clinical and biomedical applications (e.g. ElectroCardioGraphy and ElectroEncephaloGraphy) and they usually present very low skin-contact impedances and reasonable stability over the required frequency range. Hereafter, the $\mathrm{Ag} / \mathrm{AgCl}$ electrodes were used as the control electrodes. Nevertheless, IrO has been proved to be one of the most promising stimulation materials (high charge delivery capacity and low constant impedance over the entire frequency range for neural stimulation and biocompatibility) [11]. Among other deposition processes, reactive sputtering may be used in iridium oxide thin-films deposition, whether the target is DC-powered [12] or RF-powered [11] in a plasma environment.

It has been stated that skin impedance is determined mainly by the stratum corneum at frequencies below $10 \mathrm{kHz}$ [13] (see Fig. 1). Furthermore, ECG and EEG applications fall entirely into this frequency range. This outer skin layer has high-impedance characteristics since it is mainly constituted by dead skin cells and has very small water content. Consequently, biopotential electrodes require skin preparation (e.g. skin abrasion) and use of electrolytic gel to bypass the stratum corneum isolation properties and reduce interface impedance-see Fig. 1(a). Therefore and as depicted in Fig. 1(b), 


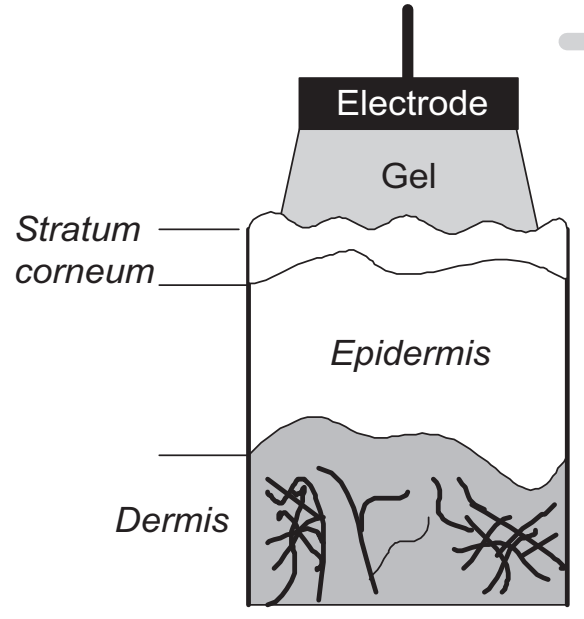

a

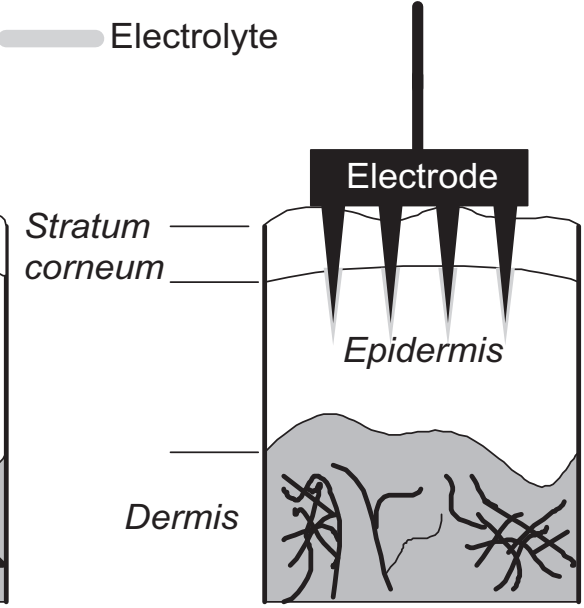

b

Fig. 1. Application of biopotential electrodes: (a) standard EEG electrode; (b) EEG electrode with microtips.

biopotential electrodes able to penetrate the outer skin layer and interface directly to the electrolytic fluids (abundance of some chlorides like $\mathrm{NaCl}$ ) of deeper tissues (e.g. living dermis) are of great interest. $\mathrm{Ag} / \mathrm{AgCl}$ has been proposed as a dry electrode coating with promising results [14]. However, the silver chloride showed to be toxic and has an associated infection risk since it dissolves on skin [15].

Each dry electrode presented in this paper is an array with $4 \times 4$ microtips covered with a thin layer of iridium oxide (IrO). The IrO coating layer was deposited by DC-sputtering technique after the microtips array bulk micromachining through a wet-etching process with undercut in a potassium hydroxide $(\mathrm{KOH})$ solution.

The design of the microtips consists in a pyramidal structure. The penetration of the microtips in the skin requires a specific pressure on the array (axial load is applied) and is given by:

$P=\frac{F}{A}$

where $P$ is the pressure resulted in the solid structure (i.e. microtip), $F$, the perpendicular force applied, and $A$, the section where the force is applied [16]. The force necessary to insert and remove the electrode is about $10 \mathrm{~N}$ [16]. Fig. 2(a) shows the pairs of actionreaction forces, established in the electrode when inserted on the skin considering, as the worst case scenarium, that skin and sil- icon have the same mechanical resistance. Assuming a uniform distribution of the force over the $4 \times 4$ microtips array $(0.625 \mathrm{~N}$ per microtip), the base of each microtip structure is subjected to a pressure of $15.6 \mathrm{MPa}$. Therefore, every microtip achieves a maximum pressure endured by silicon at $165 \mu \mathrm{m}$ from the base-red section in Fig. 2(a). Since the average height of the pyramidal structures is about $150 \mu \mathrm{m}$ and the mechanical resistance of the skin is lower than silicon's, few (or none) microtips are expected to break during skin penetration. Additionally, only $5 \%$ of the microtip electrode arrays with aspect ratios higher than the proposed electrode are expected to break [16]. As depicted in Fig. 2(b), each microtip in the array is at least $100 \mu \mathrm{m}$ high in order to pass through the stratum corneum. The fabricated dry electrodes were compared with the standard $\mathrm{Ag} / \mathrm{AgCl}$ biopotential electrodes-see Fig. 3 for comparison.

\section{Fabrication}

A wet-etch process using $\mathrm{KOH}$ was applied in the bulk micromachining $[15,17,18,19]$ of the silicon microtips. The tip shape was defined by the undercut effect in the etch process, where locally fastest-etching planes are revealed. A silicon wafer with $\langle 100\rangle$ orientation was used with a silicon nitride layer as mask for the

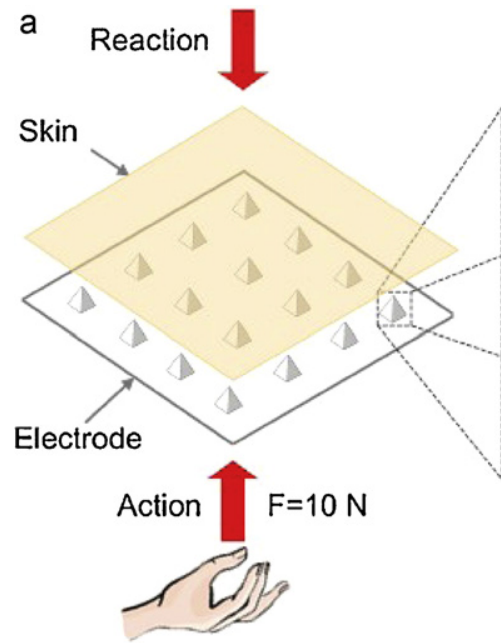

b
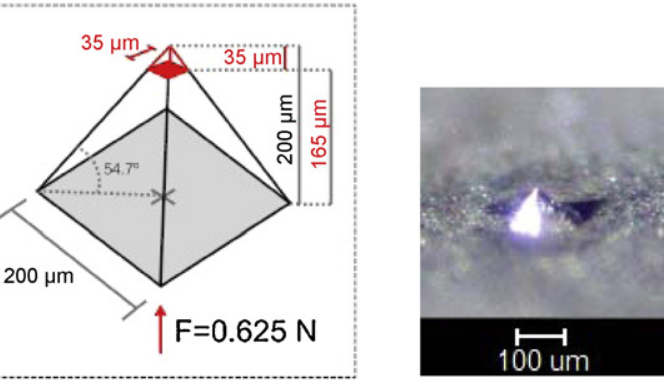

Fig. 2. (a) Pairs of action-reaction forces on a electrode with an array of $4 \times 4$ microtips; (b) magnified photograph of a microtip with pyramidal shape. 

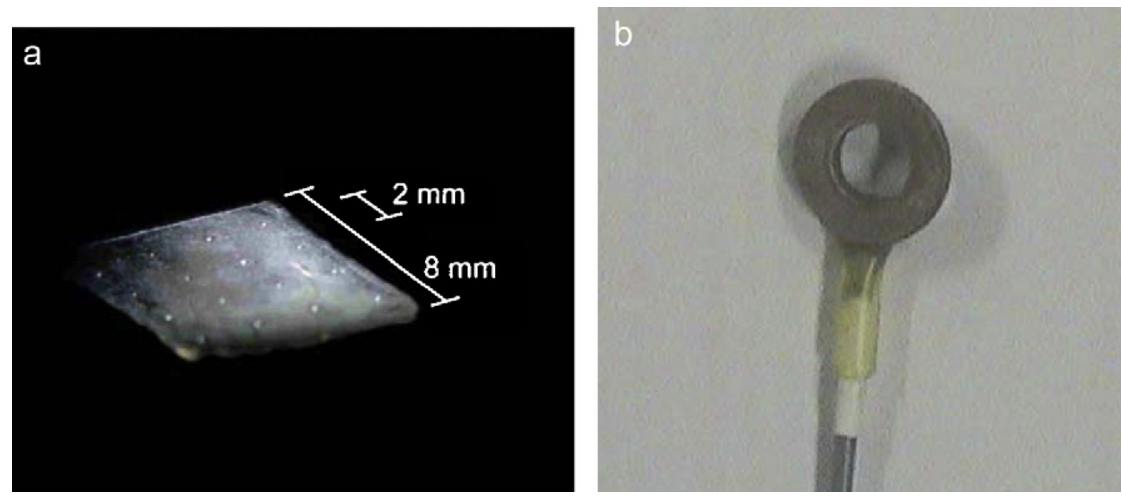

Fig. 3. (a) Optic microscope image of a micro-tips array; (b) a standard sintered $\mathrm{Ag} / \mathrm{AgCl}$ ring electrode.

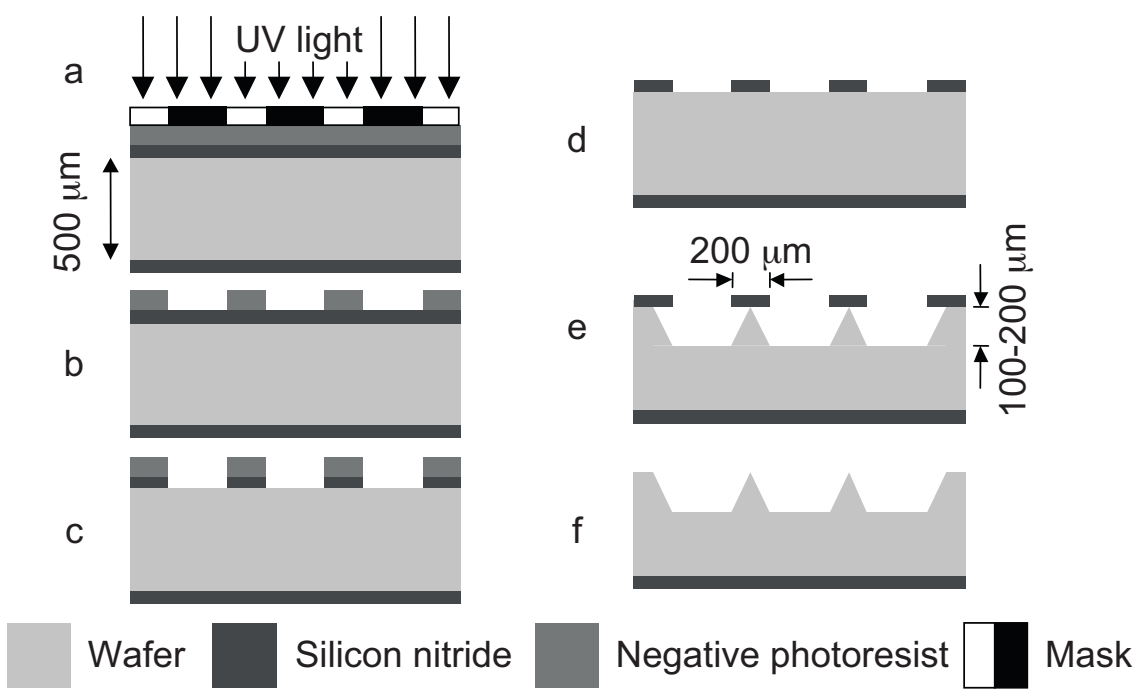

Fig. 4. The steps used in the fabrication of the microtip structures.

etching. As shown in Fig. 4, the definition of the microtips is done through a lithography process (a) in order to remove the silicon nitride from the unprotected regions (b) and (c). Then, the remaining areas of silicon nitride protects the silicon wafer during the etching process (d) and (e). The microtips emerged from the undercut effect on the etch process, which was photographed by means of optical microscopy every $30 \mathrm{~min}$ (see Fig. 5). Fig. 5 presents the evolution of the etching process from which is possible to follow the

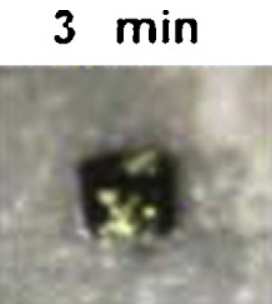

$150 \mathrm{~min}$

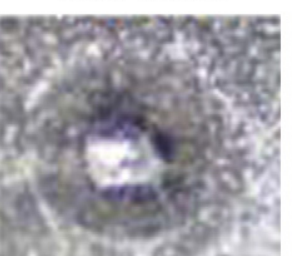

$90 \min$

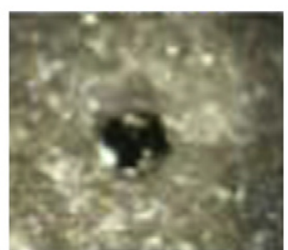

$180 \mathrm{~min}$

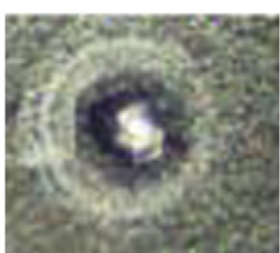

\section{$120 \mathrm{~min}$}

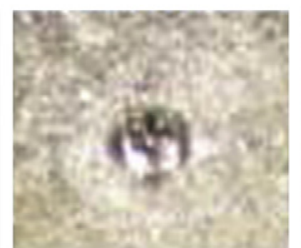

$210 \mathrm{~min}$

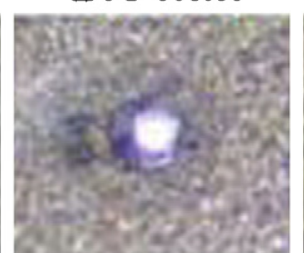

\section{$240 \mathrm{~min}$}

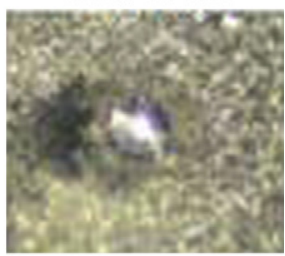

\section{0 um} $\mapsto$

Fig. 5. Top view of etching progression stages in 30 min intervals. 

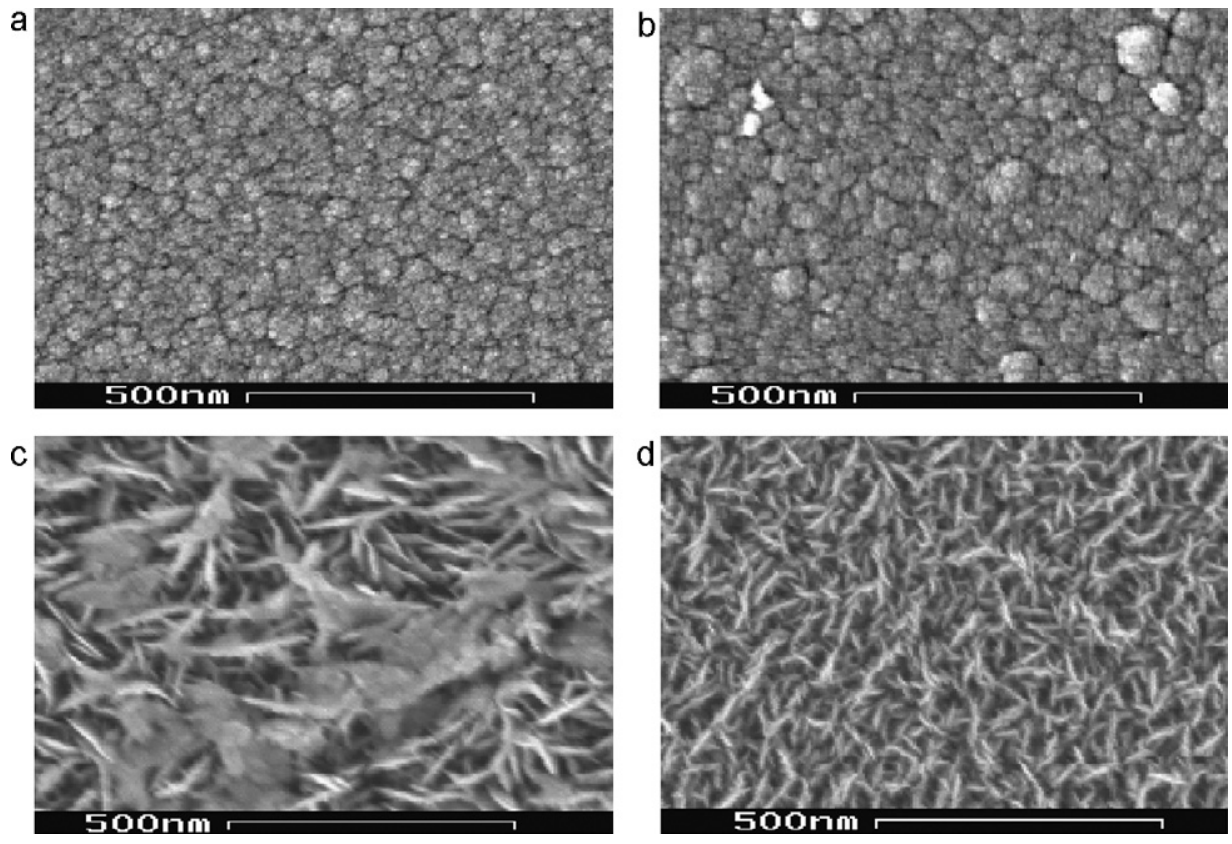

Fig. 6. SEM images of IrO thin-film surfaces deposited at different oxygen flows: (a) $2 \mathrm{sccm}$; (b) $3.5 \mathrm{sccm}$; (c) $6.5 \mathrm{sccm}$; (d) $10 \mathrm{sccm}$.

progress of the lateral plans that form a three dimensional structure. This process is anisotropic, with an angle of $54.7^{\circ}$ in the [111] plane. The pyramidal structure (Fig. 2) emerges as a result of the higher etching rate of the wafer top plane, when compared to the side plane etching rate. Actually, the final structure has a hexagonal shape base due to the anisotropic process that etched the square corner regions-see Fig. 2.

The final fabrication step of the electrodes was the deposition of a conductive IrO thin layer. The main goal of this layer was to create a conductive channel through the stratum corneum (the nonconductive skin layer) and thus to minimize the resistivity of the interface electrode/skin. The IrO is fully biocompatible, avoiding any risk to the subject. A previous sputtering of a titanium (Ti) adhesion layer was required before the IrO sputtering deposition.

In previous studies, different IrO sputtering sessions were performed onto the electrodes, all done at the same argon working gas flow ( $75 \mathrm{sccm}$ ) and DC-power ( $700 \mathrm{~W}$ ), but at different oxygen-flow rates. The analysis of the deposited thin-films surfaces revealed that an increase of oxygen flow changes the surface structure to a more grainy and fractal surface, leading to a loss of coherence and mechanical stability-see Fig. 6. The electrical resistance of the IrO layers was measured using the conventional four probe van der Pauw method [17] since it is a crucial issue for biopotential electrodes.

The lowest resistance observed along all sputtering sessions was $166.4 \times 10^{-7} \Omega \mathrm{cm}$ in a $2 \mu \mathrm{m}$ thick film for an oxygen flow of $3.5 \mathrm{sccm}$ (Fig. 6b), being the oxygen flow that was actually used on the fabricated dry electrodes. Table 1 lists the parameters for the sputtering sessions ( $\mathrm{Ti}$ and $\mathrm{IrO}$ ) of this study.

Table 1

Reactive DC-sputtering parameters.

\begin{tabular}{lll}
\hline & Sputtering session 1 & Sputtering session 2 \\
\hline Target material & $\mathrm{Ti}$ & $\mathrm{Ir}$ \\
DC Power $[\mathrm{W}]$ & 250 & 700 \\
Ar flow $[\mathrm{sccm}]$ & 55 & 75 \\
$\mathrm{O}_{2}$ flow $[\mathrm{sccm}]$ & - & 3.5 \\
Throttle & $15 \%$ & $55 \%$ \\
Temperature $\left[{ }^{\circ} \mathrm{C}\right]$ & $\mathrm{RT}$ & 200 \\
Duration $[\mathrm{s}]$ & 1080 & 3600 \\
\hline
\end{tabular}

\section{Experimental}

\subsection{Characterization}

A set of experiments were done in order to obtain the electrochemical characteristics of the electrodes as well as their interface with the electrolyte. An aqueous $\mathrm{NaCl}$ solution (most important fluid in the human tissue) with the concentration of $0.9 \%$ by weight was used as the electrolyte solution. The $\mathrm{Na}^{+}$and $\mathrm{Cl}^{-}$ions are charge carriers free to migrate in an electric field, thus contributing to DC conductivity [13]. The experimental procedure consists in a pair of electrodes submersed in the electrolyte solution of the electrochemical cell at controlled inter-electrode distances.

The electrode-electrolyte impedance (EEI) of the electrodes was estimated from the impedance spectroscopy (IS) measurements of the electrode-electrolyte-electrode system. Fig. 7 presents the measurement setup employed on IS analysis as well as the electrode-electrolyte-electrode simplified model between nodes 1 and 2 . The resistors $R_{e e 1}$ and $R_{e e 2}$ represent the resistive behavior of the electrode-electrolyte interface in each electrode. The quantities $C_{e e 1}$ and $C_{e e 2}$ represent the capacitive behavior of the electrode-electrolyte interface in each electrode. $R_{e}$ refers to the resistance of the electrolyte separating both electrodes in the electrochemical cell. Therefore, the electrode-electrolyte-electrode interface is expected to show a purely resistive behavior with low amplitude $\left(R_{e}\right)$ for high frequencies and high amplitude $\left(R_{e e 1}+R_{e}+R_{e e 2}\right)$ for low frequencies [20]. Although the electrical

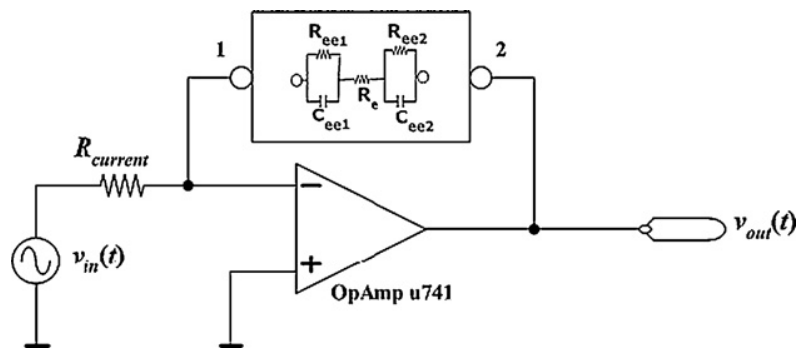

Fig. 7. Impedance-voltage converter circuit based on the operational amplifier inverter configuration. 


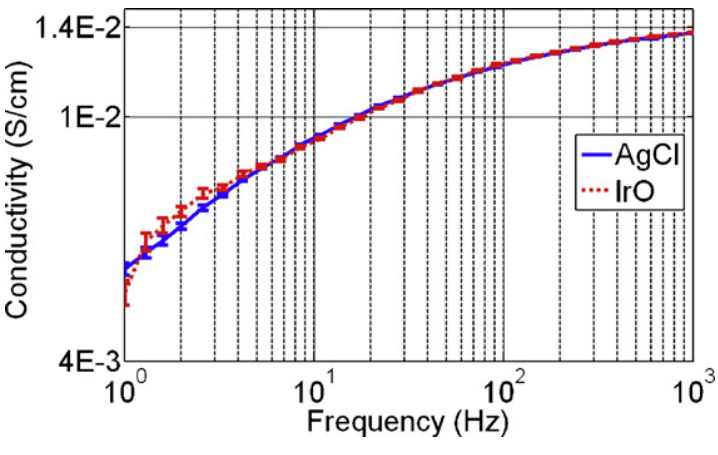

Fig. 8. Conductivity, $\sigma\left[\mathrm{Scm}^{-1}\right]$ of the fabricated IrO electrodes (red dashed line) and of the control $\mathrm{Ag} / \mathrm{AgCl}$ electrodes (blue solid line), for the whole frequency range $(1-1000 \mathrm{~Hz})$. (For interpretation of the references to color in this figure legend, the reader is referred to the web version of the article.)

model above is often assessed for planar electrodes, it should be also suitable for the microtips matrix since its IrO coating will result from a uniform sputtering material deposition thus, defining the electrochemical behavior of the electrode. Several other approaches can be used for bio-impedance spectral evaluation [15], but the preferred IS technique can use a regular PC equipped with analogue-to-digital converter (ADC) and digital-to-analogue converter (DAC) boards for digital signal generation. A composite waveform, with a finite number of sinusoidal components was used as the input signal, $V_{i n}$, on the impedance to voltage converter circuit illustrated in Fig. 7. Two PCs equipped with data acquisition (DAQ) boards were used to implement the IS measurement system. The large processing latencies spent by one PC running both signal acquisition and signal generation tasks was the reason to prefer a setup with two PCs. One PC runs an application to generate the input signal, $V_{i n}$, and the other one runs the data acquisition application to record the output signal, $V_{\text {out }}$. The resistance $R_{\text {current }}$ in the impedance conversion circuit helps to limit the current flowing through the electrochemical cell (i.e. electrode-electrolyte-electrode sample). The current that flows through the solution was kept below $100 \mu \mathrm{A}$. When $R_{\text {current }}=1 \mathrm{k} \Omega$,the DC values of $V_{\text {out }}$ were below $100 \mathrm{mV}$. The operational amplifier provides a virtual ground on its negative input which makes the impedance measurement easier.

The $V_{\text {in }}$ frequency was swept between $1 \mathrm{~Hz}$ and $1 \mathrm{kHz}$ to do the measurements. Figs. 8-10 show the conductivity, relative permittivity and impedance phase, respectively. In electrochemical analyses, the admittance values, $Y$ [S], are often assessed instead of the actually read impedance values, $Z[\Omega]$, thus, $Y=Z^{-1}$, where $Y=G+j B$ and $Z=R+j X$ ( $G$ is conductance, $B$ is susceptance,

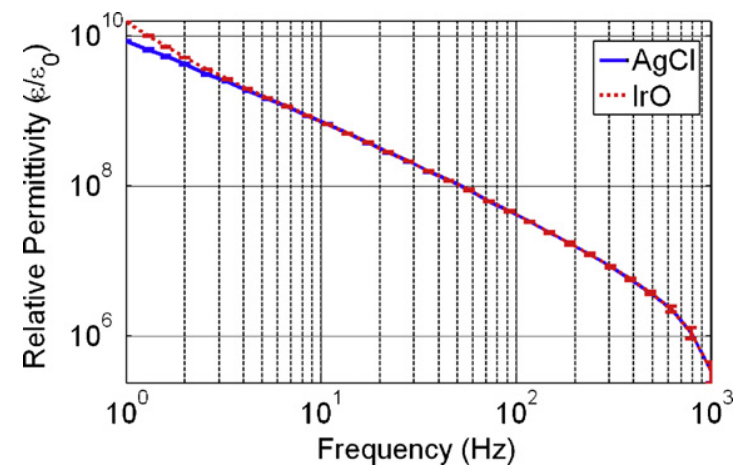

Fig. 9. Relative permittivity $\varepsilon_{r}=\varepsilon / \varepsilon_{0}$ of the fabricated IrO electrodes (red dashed line) and of the control $\mathrm{Ag} / \mathrm{AgCl}$ electrodes (blue solid line), for the whole frequency range $(1-1000 \mathrm{~Hz})$. (For interpretation of the references to color in this figure legend, the reader is referred to the web version of the article.)

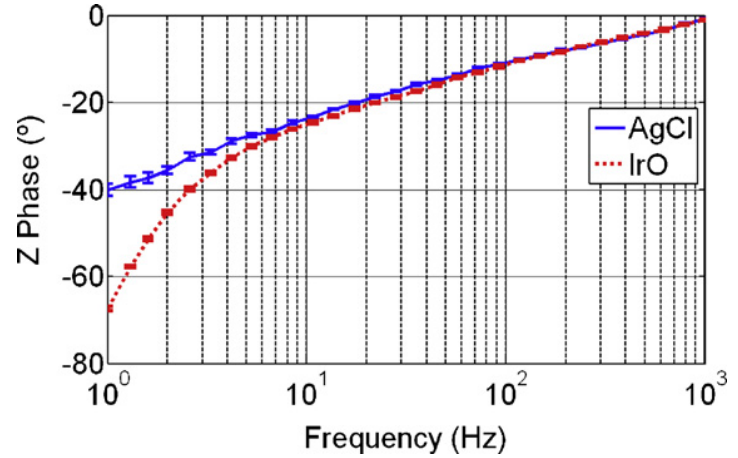

Fig. 10. Impedance phase measured for the fabricated IrO electrodes (red dashed line) and the control $\mathrm{Ag} / \mathrm{AgCl}$ electrodes (blue solid line) for the whole frequency range $(1-1000 \mathrm{~Hz})$. (For interpretation of the references to color in this figure legend, the reader is referred to the web version of the article.)

$R$ is resistance, and $X$ is reactance). The conductivity is the cellgeometry-independent value of the conductance, $G$, and usually assumed as the inverse of the resistivity (i.e. material property that is the cell-geometry-independent value of the resistance). The measurements showed that the conductivity ranges from around $5 \mathrm{mS} \mathrm{cm}^{-1}$ at $1 \mathrm{~Hz}$ to almost $14 \mathrm{mS} \mathrm{cm}^{-1}$ at $1000 \mathrm{~Hz}$. At frequencies bellow $3 \mathrm{~Hz}$, the conductivity of IrO electrodes is significantly different from that found in $\mathrm{Ag} / \mathrm{AgCl}$ electrodes. The higher permittivity exhibited by IrO electrodes for frequencies below $3 \mathrm{~Hz}$, in addition to a more negative impedance phase, mean that the IrO electrodes are more capacitive than $\mathrm{AgCl}$ electrodes for low frequencies. For frequencies above $3 \mathrm{~Hz}$, both electrodes show comparable behavior in conductivity, permittivity and phase plots. The relative permittivity, $\varepsilon_{r}$, is the cell-geometry-independent value of the susceptance, $B$, in respect to vacuum permittivity (e.g., $\left.\varepsilon_{0}=10^{-11} /(36 \pi) \mathrm{F} \mathrm{cm}^{-1}\right)$ and gives some insight about the amount of capacitive behavior of each electrode type. The $\mathrm{AgCl}$ electrodes relative permittivity ranges from $3.5 \times 10^{5}$ at $1 \mathrm{kHz}$ up to $10^{10}$ at $1 \mathrm{~Hz}$. The measurements done on frequencies below $3 \mathrm{~Hz}$ showed that the relative permittivity of IrO electrodes is slightly higher than $\mathrm{AgCl}$ electrodes. The $\mathrm{AgCl}$ electrodes presented an impedance phase of $-40^{\circ}$ for $1 \mathrm{~Hz}$ that decreases with frequency until it gets around $0^{\circ}$ for $1 \mathrm{kHz}$. The IrO electrodes manifested significantly higher impedance phase values $\left(-67^{\circ}\right.$ for $\left.1 \mathrm{~Hz}\right)$ for frequencies below $3 \mathrm{~Hz}$.

\subsection{ElectroOculoGram (EOG) experiment}

A preliminary performance evaluation of the microtips electrode with IrO coating took place during an EOG experiment. The EOG was recorded by means of electrodes laterally placed at the canthi of the eyes. As a result of the cornoretinal standing voltage potential (the cornea is positive relative to the retinal fundus), the eye movements produce changes in the potential between electrodes. The microtips array and the standard commercially available $\mathrm{Ag} / \mathrm{AgCl}$ electrodes were placed horizontally to the eyes (horizontal EOG) and both signals were recorded simultaneously. $\mathrm{Ag} / \mathrm{AgCl}$ electrodes were placed after a previous skin preparation (i.e. skin abrasion) and standard electrolytic gel was used to improve electrode-skin interface. Neither skin preparation nor electrolytic gel were applied to the electrodes with microtips. EOG data were acquired at 2000 samples/s by an EEG acquisition system with 22 bits resolution. The signals of both electrode types were digitally band-filtered $(1-40 \mathrm{~Hz})$ and a notch filter was also applied $(50 \mathrm{~Hz})$ to avoid the major interference of the electrical mains. Butterworth (zero-phase corrected) filters were employed.

The subject was instructed to sequentially look to one of two points that were horizontally equidistant from the centre of his 


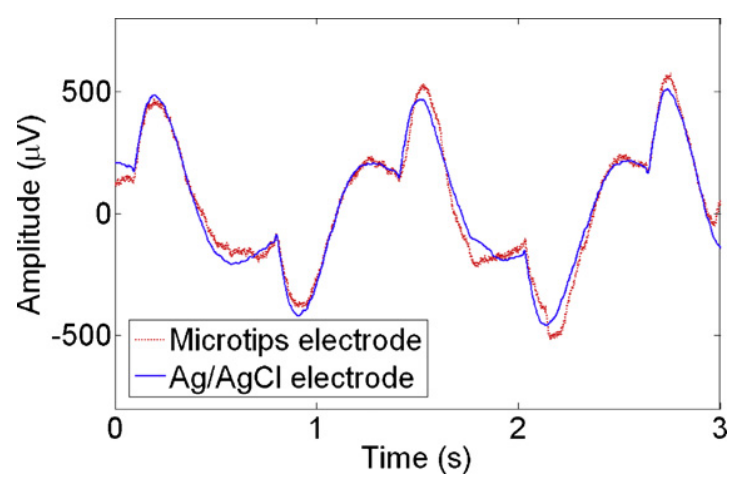

Fig. 11. Time-domain recorded signals from both the control $\mathrm{Ag} / \mathrm{AgCl}$ electrodes (blue solid line) and the microtips electrode with IrO coating (red dashed line) during an EOG experiment. (For interpretation of the references to color in this figure legend, the reader is referred to the web version of the article.)

visual field. The subject performed three eye movement trials of $1 \mathrm{~min}$ each, with $1 \mathrm{~min}$ long inter-trial period (i.e. resting period). The whole EOG session took $5 \mathrm{~min}$. Fig. 11 presents $3 \mathrm{~s}$ of the recorded EOG data for both the $\mathrm{Ag} / \mathrm{AgCl}$ electrode and the microtips electrode. The EOG data, with around $1 \mathrm{mV}$ amplitude (peak-topeak), were recorded when the subject was instructed to make eye horizontal movements. Although the $\mathrm{Ag} / \mathrm{AgCl}$ electrode was applied after a previous skin preparation and the microtips electrode directly applied on skin, both electrodes present similar signals.

\section{Discussion}

This paper presented a new dry electrode with microtips for biopotential recordings and stimulation. The new dry electrodes are intended to avoid skin preparation (i.e. electrolyte application and skin abrasion). The conductivity values suggested that the resistive behaviors of both recording materials $(\mathrm{Ag} / \mathrm{AgCl}$ and IrO) are comparable for the whole frequency range. The conductivity increases steadily until the $1 \mathrm{kHz}$ frequency limit suggesting that there is no leakage currents through the substrate for the frequency range of interest. The unequal behavior of the IrO and $\mathrm{Ag} / \mathrm{AgCl}$ materials, for frequencies below $3 \mathrm{~Hz}$, seems due to different kind of charge transmissions. The chemical reactions that assure the electron and ion exchange between non-polarizable electrodes and the electrolyte seem to occur with more extent at the $\mathrm{Ag} / \mathrm{AgCl}$ electrodes than at the IrO electrodes, for low frequencies. This is in agreement with the general knowledge that IrO coatings tend to produce polarizable electrodes (interface can be modelled by a capacitance) and $\mathrm{Ag} / \mathrm{AgCl}$ are non-polarizable electrodes. Figs. 9 and 10 illustrate the capacitive behavior of the IrO electrodes: IrO electrodes present higher permittivity (capacitance is directly proportional to permittivity) than $\mathrm{Ag} / \mathrm{AgCl}$ electrodes for low frequencies; impedance phase is more negative for IrO than for $\mathrm{Ag} / \mathrm{AgCl}$ electrodes at low-frequencies. It should be noted that the electron exchange reactions are an undesired phenomenon at minimally invasive dry electrodes that are supposed to pass through the outer skin layer. Tissue damage due to excessive accumulation of ions such as $\mathrm{Cl}^{-}$(infection risk) or even electrode degradation is more likely in these conditions. Hence, DC currents should be avoided and polarizable electrodes should be employed. In those cases, AC currents are measured through the electrical double layer that is formed at the electrode-electrolyte interface. The double layer capacitance values are dependent on the type of metal, the surface conditions, the type of electrolyte and the applied voltage. Despite the quality of the signals collected by the microtips array during the EOG experiment, the electrode-tissue interface can still be improved by increasing their surface permeability and making the surface to be dendrite structured [11]. These techniques increase the surface area of the electrode that is in contact with the electrolyte. As far as the surface area enlargement does not compromise the electrode mechanical stability, it seems to be a reliable way to decrease the electrode-tissue impedance thus, to improve the quality of the signal to be acquired (in comparison to typical EOG).

The signals recorded during the EOG experiment for both the standard $\mathrm{Ag} / \mathrm{AgCl}$ and the microtip electrodes look very similar for the horizontal eye movement task employed. These results suggest that the microtips array with IrO coating may resemble the traditional $\mathrm{Ag} / \mathrm{AgCl}$ electrodes for acquisition of biosignals, whose $\mathrm{DC}$ component can be filtered out.

From previous studies about stimulating materials, iridium oxide showed to over perform $\mathrm{Ag} / \mathrm{AgCl}$ in respect to charge delivery capacity and low-constant transition impedance [18]. IrO also demonstrated long-term mechanical stability and corrosion resistance. Therefore, considering the comparable performance of both materials for recording purposes suggested by the presented results, IrO is proposed to coat a microtip structured biopotential electrode array with recording and stimulation capabilities. The $100-200 \mu \mathrm{m}$ high microtips and the high charge delivery capacity of IrO make this electrode suitable for electrotactile stimulation applications. The ability to bypass the outer skin layer $(10 \mu \mathrm{m}$ thick) brings the electrode near the excitable nerves which decreases the potential value needed for tactile sensor stimulation. Assistive technologies for motor impaired people like brain-computer interfaces (BCIs) benefit of alternative feedback pathways to close the control loop whenever the other sensory inputs (e.g. sight and hearing) are impaired or disturbed [19].

\section{Conclusions}

This paper presented a dry IrO electrode with microtips for biopotential recordings and stimulation, which was fabricated to avoid skin preparation (i.e. electrolyte application and skin abrasion). The conductivity values suggested that the resistive behaviors of both recording materials $(\mathrm{Ag} / \mathrm{AgCl}$ and $\mathrm{IrO})$ are comparable for the whole frequency range. The unequal behavior of the IrO and $\mathrm{Ag} / \mathrm{AgCl}$ materials, for frequencies below $3 \mathrm{~Hz}$, seems due to different kind of charge transmissions.

This new dry electrode can be used simultaneously for biopotential recording (e.g. EEG, ECG, EOG and EMG) and electrotactile stimulation (e.g. mouth, forehead or arms stimulation) for use in new generation of human-machine interaction in non-restrained environments (i.e. subject senses, like sight or hearing, are exposed to random and unpredictable stimuli). Besides accomplishing both recording and stimulating tasks, the $\mathrm{KOH}$ wet-etch process is less expensive than other processes available for microtips fabrication [3]. Although the new electrode arrays present microtips with moderate aspect ratios, they seem still able to penetrate the outer skin layer and are more mechanically stable than tips with higher aspect ratios. A three-dimensional structure (microtip) with a low resistance IrO film on its surface resulted in the construction of a low-cost dry electrode for biopotential recording/stimulation that is mechanically robust and allows fast application by avoiding skin preparation (suitable to wearable and wireless applications).

\section{Acknowledgements}

This work was fully supported by FCT/PTDC/EEAENE/66855/2006 project. N.S. Dias was supported by the Portuguese Foundation for Science and Technology (SFRH/BD/21529/2005). 


\section{References}

[1] W.R. Patterson, Y. Song, C. Bull, I. Ozden, A.P. Deangellis, C. Lay, et al., A microelectrode/microelectronic hybrid device for brain implantable neuroprosthesis applications, IEEE Transactions on Biomedical Engineering 51 (2004) 1845-1853.

[2] M. Lebedev, M. Nicolelis, Brain-machine interfaces: past, present and future, Trends in Neurosciences 29 (2006) 536-546.

[3] P. Griss, P. Enoksson, Micromachined electrodes for biopotential measurements, Journal of Microelectronic Systems 10 (2001) 10-16.

[4] V.K. Mushahwar, P.L. Jacobs, R.A. Normann, R.J. Triolo, N. Kleitman, New functional electrical stimulation approaches to standing and walking, Journal of Neural Engineering 4 (2007) S181-S197.

[5] K. Kaczmarek, J. Webster, P. Bachyrita, W. Tompkins, Electrotactile and vibrotactile displays for sensory substitution systems, IEEE Transactions on Biomedical Engineering 38 (1991) 1-16.

[6] J. Mclaughlin, E. Mcadams, J. Anderson, Novel dry electrode ECG sensor system, in: Proc. Annu. Int. Conf. IEEE Eng. Med. Biol. Soc., IEEE, 1994, p. 804.

[7] W. Heuvelman, P. Helderman, G. Janssen, S. Radelaar, TiN reactive sputter deposition studied as a function of the pumping speed, Thin Solid Films 332 (1998) 335-339.

[8] A. Lopez, P. Richardson, Capacitive electrocardiographic and bioelectric electrodes, IEEE Transactions on Biomedical Engineering BM16 (1969) 99.

[9] A. Ivorra, R. Gómez, N. Noguera, R. Villa, A. Sola, L. Palacios, G. Hotter, J. Aguiló, Minimally invasive silicon probe for electrical impedance measurements in small animals, Biosensors and Bioelectronics 19 (2003) 391-399.

[10] S.F. Cogan, T.D. Plante, J. Ehrlich, Sputtered iridium oxide films (SIROFs) for low-impedance neural stimulation and recording electrodes, in: Conf. Proc. IEEE Eng. Med. Biol. Soc., 2004, pp. 4153-4156.

[11] B. Wessling, W. Mokwa, U. Schnakenberg, RF-sputtering of iridium oxide to be used as stimulation material in functional medical implants, Journal of Micromechanics and Microengineering 16 (2006) S142-S148.

[12] A. Belkind, A. Freilich, J. Lopez, Z. Zhao, W. Zhu, K. Becker, Characterization of pulsed dc magnetron sputtering plasmas, New Journal of Physics 7 (2005) 90-190.

[13] S. Grimnes, Bioimpedance and Bioelectricity Basics, Academic Press, 2000.

[14] P. Griss, H. Tolvanen-Laakso, P. Merilainen, G. Stemme, Characterization of micromachined spiked biopotential electrodes, IEEE Transactions on Biomedical Engineering 49 (2002) 597-604.

[15] T. Ferree, P. Luu, G. Russell, D. Tucker, Scalp electrode impedance, infection risk, and EEG data quality, Clinical Neurophysiology 112 (2001) 536-544.

[16] S. Henry, D.V. Mcallister, M.G. Allen, M.R. Prausnitz, Microfabricated microneedles: a novel approach to transdermal drug delivery, Journal of Pharmaceutical Sciences 87 (1998) 922-925.

[17] L.V. Pauw, A method of measuring the resistivity and Hall coefficient on lamellae of arbitrary shape, Philips Technical Review 20 (1958) 220-224.

[18] E. Slavcheva, R. Vitushinsky, W. Mokwa, U. Schnakenberg, Sputtered iridium oxide films as charge injection material for functional electrostimulation, Journal of the Electrochemical Society 151 (2004) E226-E237.

[19] A. Chatterjee, V. Aggarwal, A. Ramos, S. Acharya, N.V. Thakor, A brain-computer interface with vibrotactile biofeedback for haptic information, Journal of Neuroengineering and Rehabilitation 4 (2007) 40.

[20] M.R. Neuman, Biopotential electrodes, in: J. Webster (Ed.), Medical Instrumentation, John Wiley \& Sons, New York, USA, 1998, pp. 183-232.

\section{Biographies}

Nuno Sergio Dias (S'06) graduated in industrial electronics and computers at University of Minho, Portugal in 2004. In 2009, he obtained the PhD degree in industrial electronics from the University of Minho, Guimarães, Portugal, in collaboration with the Center for Neural Engineering at The Pennsylvania State University, State College, PA, USA. His PhD thesis was on Brain-Machine Interface Based on Biotelemetry and Dry Electrodes. He is currently pursuing a post-doc researcher at Life and Health Sciences Research Institute - ICVS, University of Minho, Braga, Portugal. He is also a member of the IEEE Industrial Electronics Society, and of the IEEE Engineering in Medicine and Biology Society.

João Paulo Carmo (S'02-M'08) was born in 1970 at Maia, Portugal. He graduated in 1993 and received his MSc degree in 2002, both in electrical engineering from the University of Porto, Porto, Portugal. In 2007, he obtained the PhD degree in Industrial Electronics from the University of Minho, Guimarães, Portugal. His PhD thesis was on RF transceivers for integration in microsystems to be used in wireless sensors network applications. Since 2008, he is an assistant researcher at the Algoritmi Center, University of Minho. He is involved in the research on micro/nanofabrication technologies for mixed-mode/RF systems, solid state integrated sensors, microactuators and micro/nanodevices for use in wireless and biomedical applications. He is also a member of the IEEE Industrial Electronics Society.

Alexandre Ferreira da Silva (S'10) graduated, in 2007, in biomedical engineering (integrated masters) with the specialization in medical electronics, at University of Minho, Braga, Portugal. Currently, he is pursuing the PhD degree in Leaders for Technical Industries, at the same institution, over the MIT-Portugal Program in the Engineering Design and Advanced Manufacturing focus-area. Between 2006 and 2007 he spent 6 months at RWTH Aachen University, Germany, studying alternative sputtering processes in order to evaluate their performance, justifying their utilization on electrodes production. In 2009, he was a visiting student for 4 months at MIT Materials Systems Laboratory. He became a student member of the IEEE society in 2010.

Paulo Mateus Mendes (M’05) graduated in 1995, and obtained his MSc degree in Electrical Engineering, in 1999, both from University of Coimbra, Portugal. He obtained his PhD degree in Industrial Electronics, from University of Minho, in 2005. Since 2006 he has been an Assistant Professor at University of Minho, and a researcher at the Algoritmi Center. He has been involved in several projects related to project, fabrication, and characterization of microantennas for wireless microsystems. He is a member of the European Microwave Association, of the IEEE Antennas and Propagation Society, and of the IEEE Engineering in Medicine and Biology Society.

José Higino Correia (S'96-M'00) graduated in physical engineering from University of Coimbra, Portugal in 1990. He obtained in 1999 a PhD degree at the Laboratory for Electronic Instrumentation, Delft University of Technology, the Netherlands, working in the field of microsystems for optical spectral analysis. Presently, he is a full professor in Department of Industrial Electronics, University of Minho, Portugal. He was the General-Chairman of Eurosensors 2003 and MME 2007, Guimarães, Portugal. His professional interests are in micromachining and microfabrication technology for mixed-mode systems, solid-state integrated sensors, microactuators and microsystems. He is also a member of the IEEE Industrial Electronics Society. 